\title{
Evaluation of SRD1000 Superconductive Reference Devices
}

\author{
Stephan Schöttl, ${ }^{1}$ Richard Rusby, ${ }^{1}$ Henri Godfrin, ${ }^{2}$ \\ Matthias Meschke, ${ }^{2}$ Valérie Goudon, ${ }^{2}$ Sebastien Triqueneaux, ${ }^{3}$ \\ Andrea Peruzzi, ${ }^{4}$ Martin J. de Groot, ${ }^{4}$ Reyer Jochemsen, ${ }^{5}$ \\ Wim Bosch, ${ }^{6}$ Yves Hermier, ${ }^{7}$ Laurent Pitre, ${ }^{7}$ Céline Rives, ${ }^{7}$ \\ Bernd Fellmuth, ${ }^{8}$ and Jost Engert ${ }^{8}$ \\ ${ }^{1}$ National Physical Laboratory, Teddington TW11 OLW, U.K. \\ ${ }^{2}$ CNRS-CRTBT, 38042 Grenoble Cedex 9, France \\ ${ }^{3}$ Advanced Technology Department, Air Liquide, 38360 Sassenage, France \\ ${ }^{4}$ Van Swinden Laboratorium, NMi, 2600 AR Delft, The Netherlands \\ ${ }^{5}$ Kamerlingh Onnes Laboratorium, Leiden Univ., 2300 RA Leiden, Netherlands \\ ${ }^{6}$ HDL Hightech Development Leiden, 2318 MP Leiden, The Netherlands \\ ${ }^{7}$ BNM-INM CNAM, 75141 Paris Cedex 03, France \\ ${ }^{8}$ Physikalisch-Technische Bundesanstalt, 10587 Berlin, Germany
}

Recently, a superconductive reference device, SRD1000, with ten reference materials has been developed to enable simplified in situ calibration of interpolating thermometers. We report on the evaluation of SRD1000 prototypes at various laboratories. The devices were built and tested by HDL and KOL, calibrated at NMi-VSL and then distributed to other partners where they were re-calibrated against the local realisations of the PLTS-2000 or laboratory scales. As a result, we obtained data on the superconductive transition temperatures, widths and shapes for four different devices. Reproducibility, sharpness of the transitions, supercooling and sensitivity to magnetic fields are evaluated. This leads to estimates of the uncertainty in determination of the transition midpoint and temperature.

PACS numbers: 07.20.Dt, 07.20.Mc, 74.62.-c, 74.70.Ad

\section{INTRODUCTION}

Temperatures down to few millikelvin are nowadays accessible to many academic and industrial research laboratories. Nevertheless, reliable and 


\section{S. Schöttl et al.}

accurate thermometry below $1 \mathrm{~K}$ is still limited and not available to the large majority of users. To provide the ultra-low temperature research community with the basis for accurate and reliable temperature measurement below $1 \mathrm{~K}$, a Provisional Low Temperature Scale, PLTS-2000,${ }^{1}$ has been defined as an equation for the melting pressure of ${ }^{3} \mathrm{He}$. However, the time and effort required for the practical realisation of the PLTS-2000 is such ${ }^{2}$ that more direct and practical means of disseminating it to final users are necessary.

In the 1970s, NIST developed and commercialised the SRM $767^{3}$ and SRM $768^{4}$ superconductive reference devices. Production has been discontinued, and a Dutch consortium led by HDL initiated the development of a new superconductive reference device, named SRD1000, ${ }^{5}$ for the range $15 \mathrm{mK}-$ $1200 \mathrm{mK}$. The SRD1000 is seen as a very effective means of dissemination of the new scale. In the EU project "ULT Dissemination", four SRD1000 prototypes were produced by HDL, tested and calibrated at KOL and NMiVSL and finally transferred to as many independent labs (CNRS-CRTBT, PTB, BNM-INM and NPL) for evaluation. The results of the evaluation are summarised in this paper.

\section{EVALUATION PROCEDURE AND MEASUREMENTS}

The SRD1000 is provided with a sensor, preamplifier and mutual inductance measurement system with digital display and an analog output. Figure 1 shows a typical temperature dependence of the dc output showing all the transitions. The individual superconductive transitions of the reference samples were observed by monitoring the analog output of the SRD1000 mutual inductance system as a function of temperature. CNRS-CRTBT and PTB were able to investigate all the superconductive transitions of the assigned prototypes. Owing to parasitic heat leaks, BNM-INM and NPL could not reach the $\mathrm{Be}$ and $\mathrm{W}$ transitions.

Temperature sweep across the transitions was performed in small temperature steps ( $0.1 \mathrm{mK}$ or less), alternated with stabilisation plateaux of 8-30 minutes to allow thermal equilibrium. To investigate the presence of hysteretical behaviour due to possible supercooling, the passage across the transitions was performed both by warming and cooling. To check for shortterm repeatability of the transitions, the selected sequences were repeated on the same cool-down (twice at BNM-INM, twice at least at PTB) or in some cases after warming up the system to room temperature (CNRS-CRTBT and NPL).

In general, these sweeps extended well into the fully normal and fully superconducting states. Each transition was then normalised to those two 


\section{Evaluation of SRD1000 Superconducting Reference Devices}

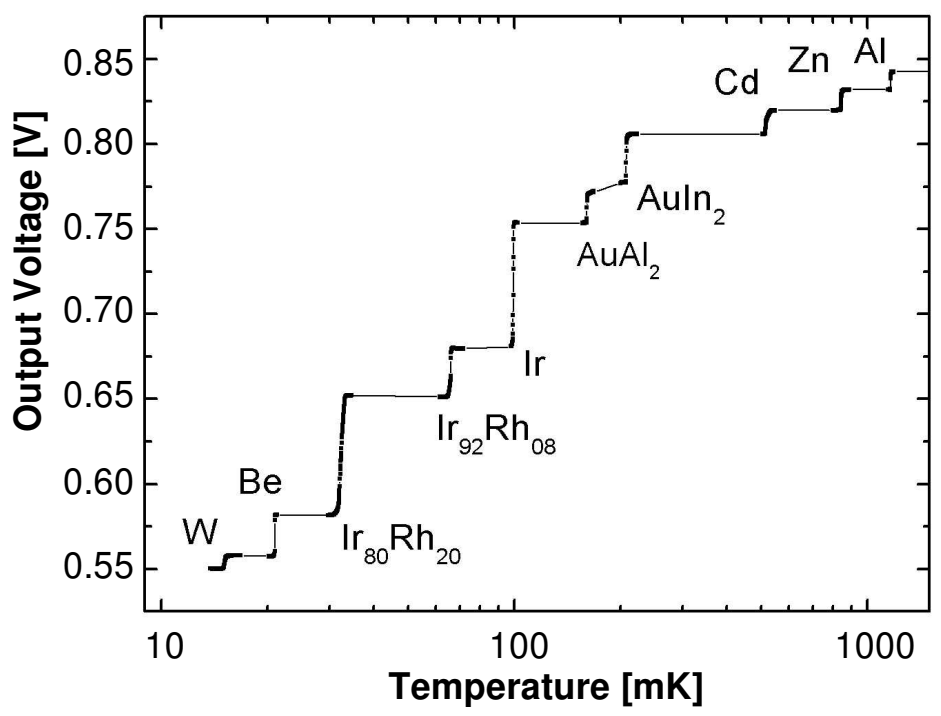

Fig. 1. Temperature dependence of SRD1000 mutual inductance as measured at the analog output.

limits, and the points of $10 \%, 50 \%$ and $90 \%$ completion of the transition were determined. ${ }^{5}$ We define the reference temperature $T_{C}$ as the midpoint (50\%), and the transition width $W_{C}$ as the full interval between the $10 \%$ and the $90 \%$ values.

\section{THERMOMETRY}

Below $1 \mathrm{~K}$, the thermometry adopted by each lab originated from the respective realisations of the PLTS- $2000^{6-8}$ or ITS-90. CNRS-CRTBT used in addition a CMN thermometer calibrated with an NBS 767 device.

An account of the uncertainties in determining the transition temperature values is given in Reference [9]. Typically, the highest contribution of the uncertainty is due to realisation of the PLTS-2000, ranging from around $0.03 \mathrm{mK}$ at CNRS-CRTBT and PTB for $T<40 \mathrm{mK}$ to around $0.2 \mathrm{mK}$ for the transitions near the melting curve minimum. At the $\mathrm{Al}$ transition, the temperature uncertainty was between $0.2 \mathrm{mK}$ (BNM-INM) and $4 \mathrm{mK}$ (CNRS-CRTBT) due to less sensitive resistance and magnetic thermometry. Adding the uncertainty in locating the transition midpoint results in total uncertainties of the absolute reference temperatures $\leq 0.2 \mathrm{mK}$ in most cases. Exceptions are $\mathrm{Cd}, \mathrm{Zn}$ and $\mathrm{Al}$, which in these prototypes undergo broader transitions than the other materials and consequently have total uncertainties between $1 \mathrm{mK}$ and $4 \mathrm{mK}$ (but see Reference [10] for later improvements). 


\section{S. Schöttl et al.}

\section{MAGNETIC FIELD TESTS}

The transition temperature $T_{C}$ of the reference samples is depressed by magnetic fields. ${ }^{2}$ For the reference materials of the SRD1000 the field dependence of $T_{C}$ is estimated to be about $-0.1 \mathrm{mK} / \mu \mathrm{T}$. Therefore, the SRD sensor is covered in two magnetic shields, made from Cryoperm and niobium, which were degaussed in situ with equipment provided by HDL.

The SRD sensors incorporate an additional, cylindrical compensation coil $^{5}$ to produce a field parallel to the device axis, and a possibility to superimpose a dc current to the planar excitation coil set (for a field component perpendicular to the device axis). Various dc currents were applied to both coil sets and the midpoint temperature monitored. Using the coil constants of the compensation coil and detector primary coil, the residual field components could be estimated and the corrections for the depression of the $T_{C}$ values could be applied.

Experiments performed at different institutes all showed similar results: The ac measuring field has an amplitude of $0.3 \mu \mathrm{T}$, which suppresses the $\mathrm{AuAl}_{2}$ transition by $22 \mu \mathrm{K}$ at most. The residual perpendicular field trapped in the shields was always less than the measuring field. The trapped field in parallel direction was determined to be $0.4 \mu \mathrm{T}$ (maximum), suppressing $T_{C}$ by less than $10 \mu \mathrm{K}$.

We concluded that the shielding is very effective and there was no need to apply a dc compensation current during subsequent measurements.

\section{RESULTS}

For each of the prototype sensors one superconductive transition is shown in Figure 2. To compare the transitions observed in different devices, the voltage output from the SRD1000 is reported as percentage of the total voltage change along the transition ( $0 \%$ corresponds to the fully superconducting state). All the $T_{C}$ and $W_{C}$ values are summarised in Table 1 .

The differences between the transition temperatures of samples of the same material reflect differences in the preparation and inhomogeneity of samples. Therefore, each device needs to be calibrated individually, in agreement with experience with SRM768. ${ }^{4}$

During an experimental run, the transition temperatures are stable well within the uncertainty of the primary thermometry and the midpoint determination. Limited information was obtained after thermal cycling to room temperature. For example, at NPL $T_{C}$ of $\mathrm{AuAl}_{2}$ and $\mathrm{Al}$ appeared to change by $0.5 \mathrm{mK}$ and $2 \mathrm{mK}$, respectively, but the second experiments were done 
Evaluation of SRD1000 Superconducting Reference Devices

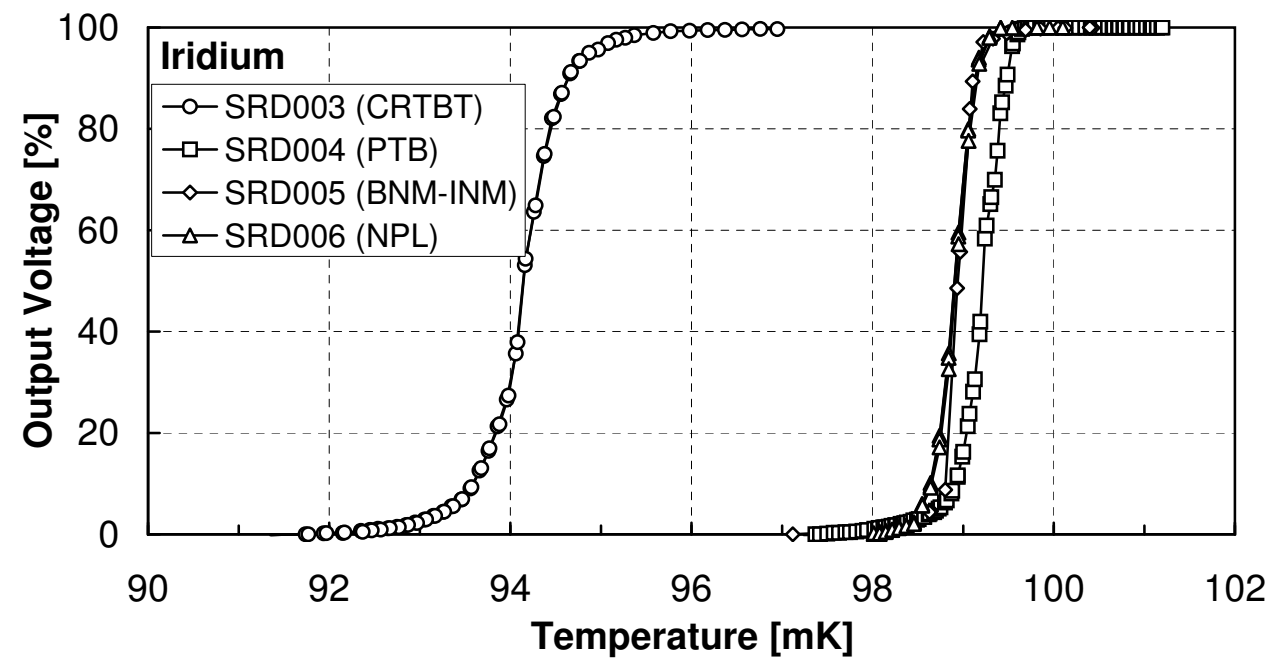

Fig. 2. Superconductive transition of Ir. SRD-003 was measured at CRTBT, SRD-004 at PTB, SRD-005 at BNM-INM, SRD-006 at NPL.

less precisely, and no firm conclusion can be drawn.

\section{CONCLUSION}

The evaluation performed on the four prototypes proved that the SRD1000 is a convenient and reliable means of transferring the PLTS-2000. The transition temperature values can be identified with an uncertainty of the order of $0.1 \%$, i.e. ranging from a few hundredths of $\mathrm{mK}$ at the lowest temperatures up to few $\mathrm{mK}$ at the highest temperatures. The residual magnetic field tests demonstrated that the magnetic shielding of the SRD sensor is very effective (attenuation factor higher than 500). As a consequence, the depression of the $T_{C}$ due to residual and measuring fields is well below the uncertainty in the scale realisation and can be neglected. Although each sample must be individually calibrated, and the long-term reproducibility of the reference points still needs further proof, the SRD1000 is a highly valuable device for high accuracy thermometry below $1 \mathrm{~K}$.

\section{ACKNOWLEDGMENTS}

This work has partly been funded by the European Commission under the Measurement and Testing activity of the Competitive and Sustainable Growth programme, contract number G6RD-CT-1999-00119. 


\section{S. Schöttl et al.}

\begin{tabular}{|c|c|c|c|c|c|}
\hline & & SRD-003 & SRD-004 & SRD-005 & SRD-006 \\
\hline$T_{C}$ & $\bar{W}$ & 15.25 & 15.20 & - & - \\
\hline \multirow[t]{9}{*}[\mathrm{mK}]{} & $\mathrm{Be}$ & 20.56 & 20.10 & - & - \\
\hline & $\operatorname{Ir}_{80} \mathrm{Rh}_{20}$ & 31.45 & 31.70 & - & 34.21 \\
\hline & $\operatorname{Ir}_{92} \mathrm{Rh}_{08}$ & 65.05 & 65.70 & 65.30 & 65.57 \\
\hline & Ir & 94.13 & 99.20 & 98.90 & 98.91 \\
\hline & $\mathrm{AuAl}_{2}$ & 137.23 & 160.60 & 160.80 & 144.95 \\
\hline & $\mathrm{AuIn}_{2}$ & 207.72 & 207.90 & 207.90 & 208.40 \\
\hline & $\mathrm{Cd}$ & 520.18 & 520.5 & 522.6 & 530.4 \\
\hline & $\mathrm{Zn}$ & 851.71 & 851.7 & 853.3 & 850.9 \\
\hline & $\mathrm{Al}$ & 1185 & 1178.2 & 1185.0 & 1178.6 \\
\hline \multirow{10}{*}{$\begin{array}{c}W_{C} \\
{[\mathrm{mK}]}\end{array}$} & $\mathrm{W}$ & 0.07 & 0.17 & - & - \\
\hline & $\mathrm{Be}$ & 0.33 & 0.03 & - & - \\
\hline & $\operatorname{Ir}_{80} \mathrm{Rh}_{20}$ & 1.17 & 1.13 & - & 0.68 \\
\hline & $\mathrm{Ir}_{92} \mathrm{Rh}_{08}$ & 0.65 & 0.94 & 0.70 & 0.57 \\
\hline & Ir & 1.07 & 0.57 & 0.30 & 0.52 \\
\hline & $\mathrm{AuAl}_{2}$ & 0.56 & 0.44 & 0.70 & 0.48 \\
\hline & $\mathrm{AuIn}_{2}$ & 1.00 & 0.65 & 0.50 & 3.20 \\
\hline & $\mathrm{Cd}$ & 12.5 & 14.5 & 12.1 & 12.6 \\
\hline & $\mathrm{Zn}$ & 9.08 & 5.42 & 15.6 & 7.8 \\
\hline & $\mathrm{Al}$ & 3.4 & 1.66 & 3.1 & 3.8 \\
\hline
\end{tabular}

Table 1. Transition temperatures $T_{C}$ and widths $W_{C}$ of the SRD prototypes.

\section{REFERENCES}

1. Proces-verbaux du Comité International des Poids et Mesures 68, 129 (2001).

2. G. Schuster, A. Hoffmann, D. Hechtfischer, "Realisation of the temperature scale PLTS-2000 at PTB", PTB-Bericht PTB-ThEx-21, ISBN 3-89701-742-3 (2001).

3. J.F. Schooley, R.J. Soulen Jr., G.A. Evans Jr., NBS Special Publication 260-44, 1-35 (1972), U.S. Govt. Printing Office, Washington D.C.

4. R.J. Soulen Jr., G.A. Evans Jr., NBS Special Publication 260-62, 1-37 (1979), U.S. Govt. Printing Office, Washington D.C.

5. W.A. Bosch et al., in Temperature: Its Measurement and Control in Science and Industry 7, 155-160 (2003), edited by D.C. Ripple, AIP, New York.

6. J. Engert, B. Fellmuth, A. Hoffmann, Journal of Low Temperature Physics 134, 425-430 (2004).

7. L. Pitre et al., in Temperature: Its Measurement and Control in Science and Industry 7, 95-100 (2003), edited by D.C. Ripple, AIP, New York.

8. A. Peruzzi, M.J. de Groot, Proc. $2^{\text {nd }}$ Int. Seminar on Low Temperature Thermometry, 35-40 (2003), edited by Szmyrka-Grzebyk et al., Wrocław, Poland.

9. A. Peruzzi et al., submitted to Proc. $9^{\text {th }}$ Int. Symposium of Temperature and Thermal Measurements (TEMPMEKO2004), Dubrovnik, Croatia.

10. W.A. Bosch, J.J.M. van der Hark, J. Pöll, R. Jochemsen, in these proceedings. 